\title{
GEBURTSVORGANG BEI MYOTIS MYOTIS (BORKHAUSEN，1797) UND ANSCHLIESSENDES VERHALTEN VON MUTTER UND JUNGEM
}

von

\section{ANTON KOLB}

\author{
Biologisches Institut, Phil. Theol. Hochschule, Bamberg
}

\begin{abstract}
Wegen der versteckten Lebensweise der Fledermäuse konnte der Geburtsvorgang und das anschließende Verhalten der Jungen noch kaum vollständig beobachtet werden. Das Bestreben, diese Lücke zu schließen, währt schon ziemlich lange. Dies ist uns im Sommer 1965 geglückt. Durch regelmäßige Kontrollen der Wochenstube des Mausohrs auf der Martinskirche in Bamberg, insbesondere im Zeitabschnitt der Geburt, haben wir ein Tier gerade bei Geburtsbeginn überrascht. Es wurde mitgenommen und in einen Versuchsschrank für Fledermäuse gesetzt, wo für diese Beobachtung bereits alle Vorbereitungen getroffen waren. Der Versuchsschrank, eine Holzkonstruktion mit den Ausmaßen $2 \times 1 \times 1 \mathrm{~m}$ ist allseitig, mit Ausnahme des Bodens, mit Glaswänden versehen. An den Ecken, an mehreren Mittel- und Deckensprossen, insbesondere aber an einer etwa 22 $\mathrm{cm}$ breiten, mit Sägeschnitten versehenen Bretterwandung, ist den Tieren die Möglichkeit zum Aufhängen gegeben.
\end{abstract}

\section{GEBURTSVORGANG}

Am 15.6.1965 um 14.50 Uhr fiel bei einer Kontrolle der obengenannten Wochenstube ein Tier dadurch auf, daß es als einziges sitzen blieb, während der kleine Klumpen durch Abfliegen sich auflöste. Entgegen der üblichen Gewohnheit der Tiere, nur mit den Hinterbeinen sich aufzuhängen, hatte es sich auch mit den Daumenkrallen angeheftet. Bei seiner Abnahme mit der Hand zeigte sich, daß die Geburt bereits im Gange war, weshalb es eiligst in das Institut gebracht wurde.

Aus der Vulva (Abb. 1) ragte bereits ein Handgelenk mit Daumenkralle und ein Knäuel der Flughaut heraus. Die Brustdrüsen waren prall angeschwollen, der Hof um die Zitzen war haarfrei. Im Versuchsschrank lief das Tier anfangs unruhig hin und her, flog dazwischen auch ein Stückchen, bis es sich schließlich neben einem bereits vorhandenen, trächtigen Weibchen derselben Art aufhing, das ebenfalls Anzeichen der herannahenden Geburt erkennen ließ. Beide Tiere hefteten sich auch hier mit den Daumenkrallen an der Unterlage an.

Um 15.20 Uhr konnte man am Hinterleib Wellen kontrahierender Muskelbewegungen feststellen, wobei der Schwanz von nun an bei beiden Tieren bogenförmig dorsalwärts gehalten wurde (Abb. 2). Die seitliche Ausbuchtung der Bauchregion verschwand. Zwischendurch hob sich ein Tier etwas hoch, führte den Kopf bauchwärts und beleckte die Vulva bzw. die bereits daraus hervorragenden Teile des Jungen. Beide Tiere vollführten dies in der gleichen Weise.

Gegen 15.38 Uhr erfolgte dann ohne merkliche Preßbewegungen plötzlich die Geburt bei dem zuletzt eingebrachten Tier. Mit dem Kopf voran wurde das Junge innerhalb weniger Sekunden aus der Vagina geschoben. Dieses war nackt und blind und krabbelte sofort auf der Bauchseite der Mutter, die sich etwas von der Unterlage abhob, nach unten, in Richtung zur Brust, und suchte, mit dem Kopf tastende, seitliche Bewegungen ausführend, nach einer Zitze (Abb. 3). Hierbei kam es an der rechten Brustzitze vorbei bis in die Kopfregion des Muttertieres, wich wieder zurück, suchte weiter, geriet auf die linke Seite und saugte sich schließlich dort an der Brustzitze fest. Die Mutter unterstützte zuletzt die Suchaktion des Jungen dadurch, daß sie mit ihrer Schnauze den Kopf des Jungen zu ihrer Brustzitze schob. Das Junge saß nun unter dem linken Flügel der Mutter. Der bis nach Austritt des Jungen aus der Scheide hochgehobene Schwanz wurde wieder in die Normallage gebracht.

Nun begann die Mutter das Junge eifrigst zu belecken. Beide waren jedoch noch durch die rötlich gefärbte Nabelschnur verbunden. Noch während die Mutter das Junge beleckte, schickte sich dieses an, sich mit den Hinterbeinen seitlich von ihr zu hängen, wobei es die Brustzitze im Maul behielt. Dadurch 
wurde die Nabelschnur zwischen der Mutter und dem Jungen straff gespannt. Um $15.50 \mathrm{Uhr}$ war die Nabelschnur merklich verblaßt. Die Anstrengungen des Jungen, sich mit dem hinteren Teil seines Körpers noch weiter seitlich zu hängen, gingen unvermindert weiter. Mit dem rechten Hinterbein stemmte es sich dabei um 16.18 Uhr am linken Unterarm der Mutter $a b$, mit dem linken Fuß krallte es sich an die Wand und zog. Die Nabelschnur nahm dadurch um etwa $1 \mathrm{~cm}$ an Länge $\mathrm{zu}$ und wurde immer blasser und dünner. Das Junge begann um 16.35 Uhr mit der hinteren Körperpartie sich seitlich noch mehr in Kopfrichtung $\mathrm{zu}$ bewegen, wodurch die Nabelschnur laufend stark gespannt blieb. Auch hierbei drückte es sich mit dem rechten Hinterbein, dessen Fuß im Fell der Mutter verkrallt war, von ihr ab, während es mit dem linken $F u ß$ an der Wand laufend weiter ausgreifend die hintere Körperpartie von der Mutter wegzuziehen versuchte. Die Nabelschnur war inzwischen dunkelbraun bis schwärzlich geworden. Die Verfärbung begann in der Mitte der Nabelschnur und setzte sich nach beiden Enden fort.

Um 17.35 Uhr unterbrach das Junge sein bisheriges Verhalten für 10 Minuten. Es ließ mit dem linken Hinterfuß die Wand los und klammerte sich an das Fell der Mutter. Aber schon um 17.45 Uhr griff es mit dem linken $\mathrm{Fuß}$ erneut nach der Wand und zog energisch (Abb. 4). Die Situation blieb unverändert bis 19.05 Uhr. Von diesem Zeitpunkt an konnte man kräftigè, ruckweise Abrückungsversuche beobachten, die laufend heftiger wurden und um 19.45 Uhr mit dem Abreißen der Nabelschnur an der Mutter endeten. Sofort rückte das Junge, an dem noch die Nabelschnur herunterhing, mit dem bisher abgestemmten hinteren Körperteil an die Seite der Mutter heran. 5 Minuten später, um 19.50 Uhr wurde die Nachgeburt ausgestossen; die Mutter führte den Kopf zur Bauchseite und fraß sie auf. Gegen 20.00 Uhr begann sie das Junge eifrig auf der Bauchseite zu belecken, wobei sie die inzwischen vollkommen vertrocknete und schwarzgewordene Nabelschnur unmittelbar am Jungen abbiß. Die Nabelschnur fiel zu Boden und damit war der Geburtsvorgang beendet.

Das zweite trächtige Weibchen verhielt sich anfangs genauso wie eben geschildert. Zur gleichen Zeit, als die beschriebene Geburt vor sich ging, vollzog sich bei ihm eine Totgeburt. Anschließend wich das Verhalten dieses Tieres von dem des anderen ab. Es verließ seine Hängelage, lief auf dem Boden herum, wobei es das an der Nabelschnur hängende tote Junge solange nachschleifte, bis dieses an einem auf dem Boden liegenden Ast hängen blieb und schließ- lich auch die Placenta ausgestoßen wurde. Die Nabelschnur wurde hierbei sehr stark in die Länge gezogen. Solche Totgeburten mit Nabelschnur und Placenta fand ich einige Male auf Dachböden verschiedener Gebäude mit Wochenstuben des Mausohrs.

\section{VERHALTEN NACH DER GEBURT}

Das Junge krabbelte anschließend an der Mutter etwas herum und kam um 20.10 Uhr auf deren Rücken zu sitzen, wo es Tick-Laute von sich gab. Mit dem rechten Arm drückte die Mutter daraufhin das Junge etwas zur Seite, faßte es leicht mit den Zähnen und zog es noch ein Stückchen weiter weg. Dann bewegte sich die Mutter etwa $15 \mathrm{~cm}$ von der bisherigen Aufhänge-Stelle weg, kehrte jedoch sofort wieder zurück und beleckte das Junge eifrig auf dem Rücken. Um 20.30 Uhr kletterte die Mutter ganz nahe an das Junge heran, lüftete den linken Flügel etwas, worauf dieses sofort an die Mutter kletterte und die Zitze suchte. Das Junge saß nun unter dem linken Flügel der Mutter, wobei es die linke Zitze im Maul hielt und mit den Hinterfüßen und den Daumenkrallen sich am Fell anheftete. Nach einer kurzen Beobachtungspause von 21-22.05 $\mathrm{Uhr}$ wurde das Junge auf der rechten Seite der Mutter gefunden.

Auch am nächsten Tag, den 16.6.65, um 7.45 Uhr wurde das Junge auf der rechten Seite angetroffen. Doch saß es schon um 7.50 Uhr wieder auf der linken Seite, während es um 8.30 Uhr wieder auf der rechten Seite hing. Dieser Lage-Wechsel am Muttertier konnte auch weiterhin beobachtet werden. Er erfolgte unregelmäßig und wurde zum Teil aktiv von der Mutter angeregt, indem sie das Junge mit der Schnauze von der Zitze, die es gerade im Maul hielt, wegdrückte, zum Teil aber auch spontan vom Jungen ausgeführt. Am vierten Tag wurde das Junge frei neben der Mutter hängend angetroffen. Wurde der Hängeplatz gewechselt, so wurde das Junge sowohl beim Klettern als auch beim Flug mitgenommen.

Mit einem Ultraschallmikrofon wurden am 1.7.65 die Tick-Laute des Jungen aufgenommen. Das Oscillogramm dieser Impulse mit seinem ungleichmäßigen Amplitudenverlauf zeigte den Vespertilioniden-Typ. Der Impuls enthielt jedoch noch Komponenten der 1. und 2. Oberwelle. Der von meinem Assistenten, nicht aber von mir noch hörbare TickLaut hatte eine Frequenz von etwa $17 \mathrm{kHz}$. Demnach hatte die 1 . Oberwelle eine Frequenz von 34 $\mathrm{kHz}$ und die 2. eine solche von $51 \mathrm{kHz}$, da die erste Oberwelle das Doppelte und die zweite das Dreifache der Grundschwingung beträgt. Zu diesem Zeit- 


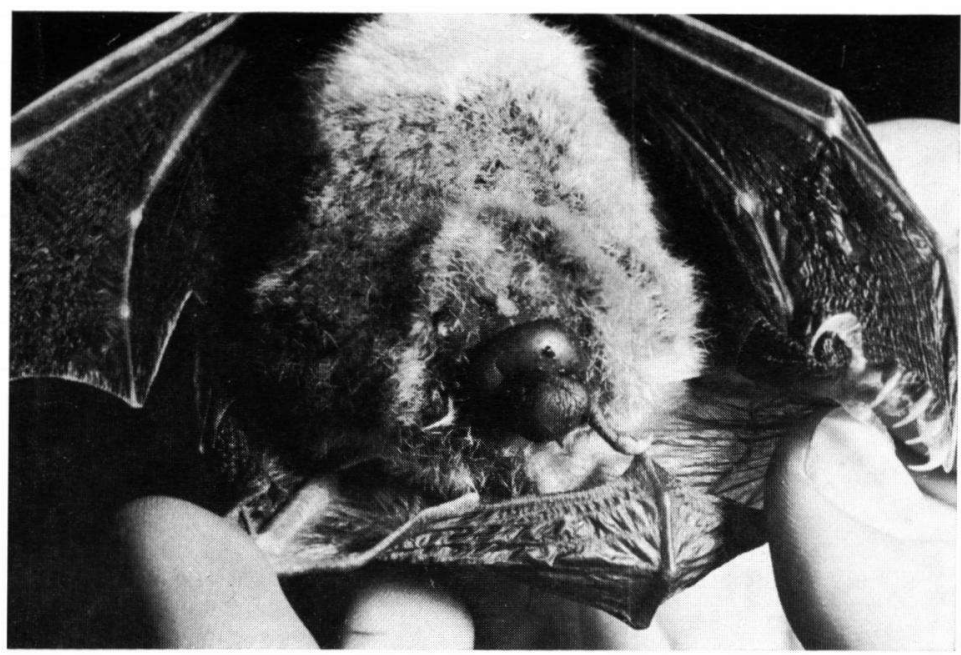

Abb. 1. Myotis myotis - Weibchen zu Beginn des Geburtsvorganges.

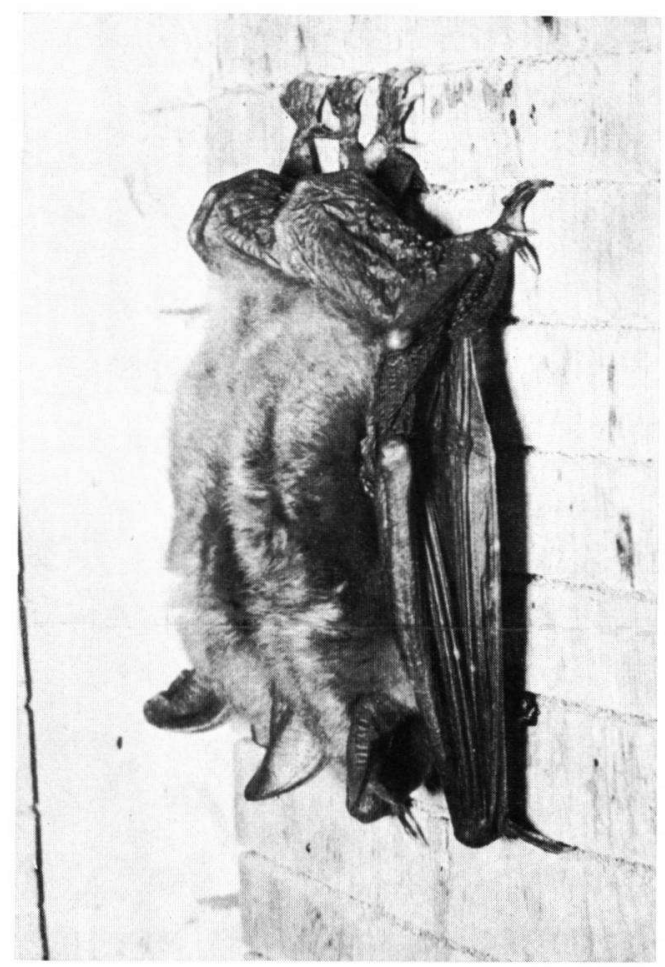

Abb. 2. Zwei Weibchen von Myotis myotis in typischer Stellung unmittelbar vor der Geburt.

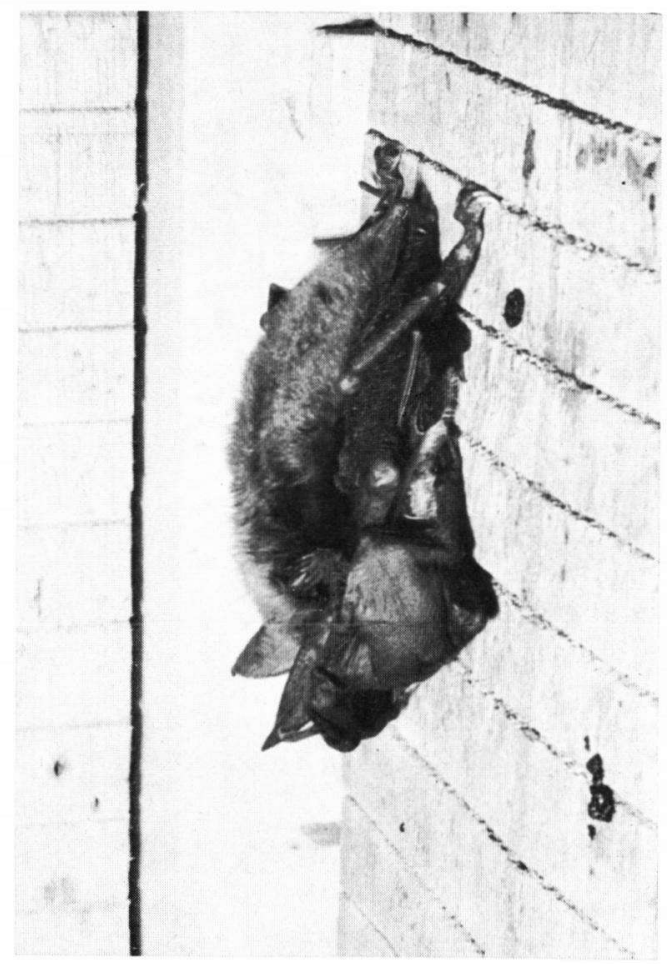

Abb. 3. Myotis myotis - Weibchen mit Jungem bei der Suche nach der Brustzitze. Die Nabelschnur ist hierbei gespannt. 


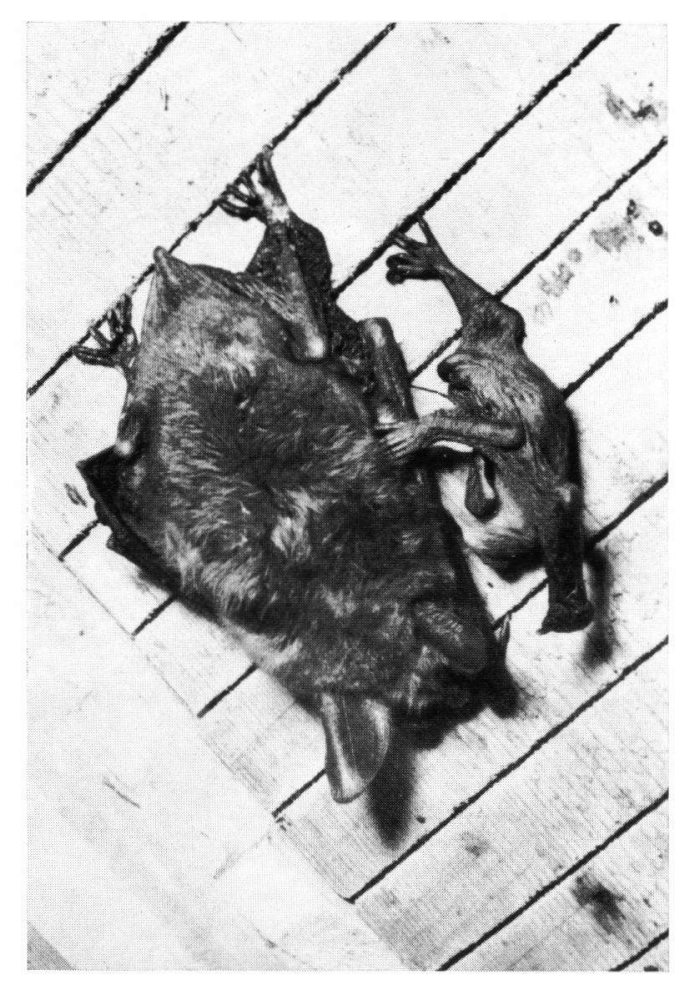

Abb. 4. Myotis myotis - Weibchen mit an der Nabelschnur zerrendem Jungen, wobei dieses dauernd die Brustzitze im Maul behält.

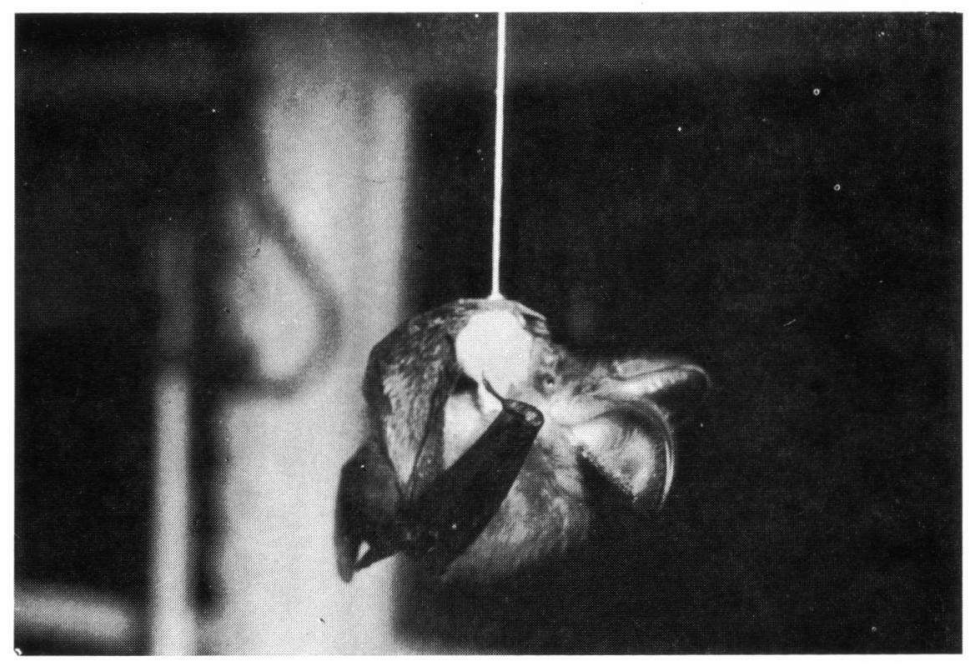

Abb. 5. Plecotus auritus - benützt beim Anhängen an einen Wattebausch bei einem Test auch den Schwanz bzw. das Uropatagium. 
punkt war das Junge, das sich sehr langsam entwickelte, noch blind. Erst am nächsten Tag, also am 16. Tag nach der Geburt, wurde es sehend.

\section{DISKUSSION}

a. Stellung des Muttertieres bei der Geburt. Uber die Stellung des Muttertieres bei der Geburt gibt es eine Reihe von Beobachtungen.

BREHM (1912) gibt den Beobachtungen von Blasius und Kolenati zufolge an, daß sich das Weibchen gegen seine Gewohnheit auch mit den Daumenkrallen aufhängt, Schwanz und Flughaut auf den Bauch schlägt und damit einen Sack bildet, in den das Junge bei der Geburt fällt. Demnach dürfte er wohl annehmen, daß sich das Weibchen bei der Geburt umdreht, zumindest jedoch, daß die vordere Körperpartie höher hängt als die hintere.

Eiseñtraut (1936) beschreibt die Geburt bei Plecotus auritus und schildert, daß sich dieses mit den Daumenkrallen und den Füßen an der Decke eines Gazekäfigs so aufhing, daß es eine horizontale Körperlage einnahm. Dabei waren die Beine gespreizt und das Ende der gespannten Schwanzflughaut mit dem Schwanz war taschenartig auf die Bauchseite geschlagen.

DitTrich (1958) berichtet, daB ein Weibchen von Nyctalus noctula während einer Totgeburt senkrecht hing, und zwar mit dem Kopf nach unten.

Eine zusammenfassende Darstellung dieses Fragenkomplexes verdanken wir WimsatT (1960), der eine große Zahl von Berichten gegeneinander abwog und zu dem Schluß kam, daß wohl das Beihalten der Ruhelage bei der Geburt das normale Verhalten darstellen dürfte und nur bei Störungen eine andere Lage eingenommen wird. Freilich gab er ganz allgemein zu bedenken, daß die Beobachtungen von Tieren in Gefangenschaft gemacht wurden. Wohl änderte auch das hier beobachtete Mausohr beim Geburtsvorgang seine sonstige Aufhängegepflogenheit, sich nur mit den Hinterfüßen aufzuhängen, insofern, als es sich auch mit den Daumenkrallen an der Unterlage anheftete, wodurch sein Körper dieselbe Richtung bekam wie die Unterlage, z.B. die Neigung des Daches. Doch brachte es sich nicht in die waagrechte Lage und es drehte sich auch nicht um. In der geschilderten Stellung, mit dem Kopfe nach unten und mit den Daumenkrallen an den Brettern des Schieferdaches festgekrallt, wurde das beschriebene Weibchen bei bereits begonnener Geburt auf dem Dachboden angetroffen. Im Versuchsschrank, der schon seit mehreren Jahren zu Fledermausversuchen verwandt wird, und möglichst natur- getreu eingerichtet ist, wählte das Tier anstelle der Schräglage des Daches die Senkrechte, obwohl es die Möglichkeit gehabt hätte, sich anders aufzuhängen. $\mathrm{Da}$ zu gleicher Zeit ein weiteres Tier dasselbe Verhalten zeigte, dürfte diese Stellung die normale sein. Wir können somit feststellen, da $B$ das Mausohr bei der Geburt die typische Stellung der Fledermäuse, den Kopf nach unten, beibehält, sich aber auch mit den Daumenkrallen an der Unterlage festheftet und somit, je nach den Verhältnissen, eine senkrechte oder einen spitzen Winkel bildende Körperlage einnimmt. Hierbei wird der bogenförmig gekrümmte Schwanz mit der Flughaut hochgehoben, wodurch ein Raum für das Junge unmittelbar nach dem Ausstoßen entsteht, der oben abgeschlossen ist.

Dieselbe senkrechte Stellung nahm auch Eptesicus serotinus (Schreber, 1774) in Gefangenschaft ein. Dieses Tier hatte in einem $20 \mathrm{~m}^{2}$ großen Raum, in dem ein kleines Holzdach errichtet war, die Möglichkeit, die senkrechte Stimseite oder die Schräge des Daches zu wählen. Es wählte die senkrechte Stimseite. Doch brachte es leider ein totes Junges zur Welt.

Die unterschiedlichen Beobachtungen über die Stellung des Muttertieres sind sicherlich richtig. Obwohl die Möglichkeit besteht, daß sich die einzelnen Arten, ja sogar Individuen, verschieden verhalten, dürte das dort geschilderte Verhalten trotzdem abgewandelt sein. So ist es einem Tier unmöglich, sich zum Beispiel von der Wand eines Gazekäfigs wegen deren Biegsamkeit abzuheben. Es muß sich daher umdrehen, um dem Jungen den Weg zur Zitze freizumachen.

Schließlich findet das Uropatagium bei den Fledermäusen eine recht unterschiedliche und vielseitige Verwendung. So fungiert es beim Flug als Steuerung und beim Erwerb von Insekten als Fangnetz. Darüber hinaus werden jedoch auch nicht mundgerecht erfaßte Beutetiere bei der Nahrungsaufnahme vom Boden in die Schwanzflughaut gestoßen, wobei diese als Widerlager dient. Im Extremfall kann die Schwanzflughaut sogar zum Festhalten an einer Unterlage verwendet werden, wie dies aus der Abb. 5 hervorgeht. Trotz der Fähigkeit des Uropatagiums, auch bei der Geburt eine zweifelsohne sichere Tasche für das Junge $z u$ bilden, muß die von vielen Forschern berichtete, veränderte Haltung eines Weibchens, wobei dieses sich entweder mit dem Kopf nach oben ausrichtet oder sich mit den 4 Extremitäten waagrecht an die Decke hängt, als abgewandelt betrachtet werden. Sie stellt eine durch die Gefangenschaft bedingte Anpassung an die veränderten Ver- 
hältnisse dar, wie dies bereits WIMSATT andeutete.

Auch die normale Stellung der Mutter mit dem Kopfe nach unten läßt dem Jungen genauso sicher die Brustzitze erreichen, wie die für menschliche $\mathrm{Ge}$ sichtspunkte noch sicherer anmutende Tasche der Schwanzflughaut bei umgekehrter Stellung. Denn nach oben wird, wie aus Abb. 2 hervorgeht, der Bewegungsbereich des Jungen durch die Schwanzflughaut mit dem bogenförmig gehobenen Schwanz abgeschlossen. Das blinde Junge jedoch beginnt, noch nicht einmal vollständig der Vagina entschlüpft, sich zu bewegen und nach der Zitze zu suchen, eine Instinkthandlung, die es ihm offensichtlich nicht erlaubt, den Körper der Mutter zu verlassen. Diese aber kann durch geringfügiges Abheben ihres Körpers von der Unterlage, wobei noch die Möglichkeit besteht, Hinter- und Vorderpartie des Körpers ungleich abzuheben, ihm den Weg frei und ungefährlich gestalten. Im übrigen muß die Tatsache, daß das Junge auch nach dem Ausstoßen durch den Nabelstrang noch fest mit der Mutter verbunden ist, als eine Art "biologischer Sicherung" angesehen werden. Denn der Nabelstrang ist fest und würde das Junge bei einem evtl. Fall ohne weiteres halten.

\section{b. Verhalten des Jungen.}

Lückenlos konnte auch das Verhalten des Jungen unmittelbar nach dem Ausstoßen beobachtet werden. Durch das Klettern des Jungen in die Brustregion der Mutter, dürfte die nach STARCK (1957) normal 18-25 $\mathrm{mm}$ lange Nabelschnur bereits etwas in die Länge gezogen werden. Noch mehr in die Länge gezogen wird diese jedoch durch das seitliche Hängen des Jungen. Dieses Phänomen des seitlichen Aufhängens des Jungen konnte schon vor mehreren Jahren (KoLB, 1954) erstmalig beobachtet, jedoch nicht lückenlos verfolgt werden. Auch die Art und Weise des seitlichen Hängens ist den Verhältnissen angepaßt und variiert. Während bei der erstmaligen Beobachtung dieses Verhaltens das Neugeborene sich am engmaschigen Deckengitter seitlich von der Mutter aufhing, da keine andere Möglichkeit bestand, benutzte es hierzu in diesem Falle die senkrecht stehenden Wandbretter. Hierbei hing es nicht in gleicher Höhe wie die Mutter.

Die zweite Stellung, seitlich von der Mutter doch etwas tiefer hängend, dürfte die normale sein. Eigenartig ist außerdem, daß bei allen bis jetzt beobachteten Geburtsvorgängen das Junge die linke Seite der Mutter zum Seitlich-Hängen auswählte. Der Sinn des seitlichen Hängens des Jungen besteht sicherlich darin, das Kollabieren der Gefäße der Nabelschnur zu bewerkstelligen und diese zum Abreißen zu bringen. Daß es wichtig ist, daß vor dem Abreißen der Nabelschnur die Gefäße ihre Funktion eingestellt haben, berichtet WIMSATT, der beobachten konnte, daß durch das Abreißen der Nabelschnur oder deren künstlicher Durchtrennung unmittelbar nach dem Ausstoßen des Jungen dieses verblutet. Uberraschend ist die eigene Aktivität des Neugeborenen. Durch das seitliche Hängen wird die Nabelschnur dauernd gespannt und schließlich durch kräftiges Zerren an der Placenta abgerissen. Wird die Placenta vor dem Abreißen der Nabelschnur ausgestoßen, so beißt das Muttertier diese beim Fressen der Placenta $a b$, wie eine Beobachtung zeigt. Das durch die Mutter vollzogene anschließende Abbeißen der Nabelschnur am Neugeborenen macht meine frühere An. nahme, daß sie dort abbrechen würde, hinfällig. Bei keinem anderen Säugetier konnte man bis jetzt beobachten, daß das Junge selbst die Durchtrennung der Nabelschnur vollzieht, weshalb diesem wohl eine gewisse Bedeutung zukommt. Die Auflösung dieser "biologischen Sicherung" wird erst vollzogen, wenn das Junge sicheren Halt an der Zitze gefunden und das Kollabieren der Gefäße in der Nabelschnur sich vollzogen hat. Die Tatsache, daß die Gefäße auch nach dem Ausstoßen des Jungen noch kurze Zeit funktionsfähig sind, unterstreicht die Bedeutung der noch bestehenden Verbindung.

\section{c. Die Nabelschnur}

Die Zeit, die das Junge zum Abreißen der Nabelschnur benötigt, variiert wohl sehr stark. Bei meiner erstmaligen Beobachtung dauerte dieser Vorgang zweieinhalb Stunden, während er dieses Mal vier Stunden und zwanzig Minuten in Anspruch nahm. Dementsprechend ist auch die Nabelschnur mehr oder weniger stark in die Länge gezogen und weist einen unterschiedlichen Grad der Vertrocknung auf. Bei Kotuntersuchungen (KoLB, 1958) habe ich Nabelschnüre mit einer Länge von $10 \mathrm{bis} 41 \mathrm{~mm}$ gefunden. Die längsten Nabelschnüre, etwa von $30-41 \mathrm{~mm}$, waren sehr dünn, vollständig vertrocknet und wiesen mitunter an einem Ende noch Fetzen der Placenta auf. Die kürzeren Nabelschnüre dagegen waren bedeutend dicker und weniger vertrocknet. $\mathrm{Da}$ auch die bei der beschriebenen Geburt angefallene Nabelschnur auf einer Seite kleine Fetzen der Placenta aufwies, sehr dünn, $42 \mathrm{~mm}$ lang und vollkommen vertrocknet war, so läßt sich daraus ein Rückschluß auf die Dauer des Geburtsvorganges an Hand des Zustandes der Nabelschnur ziehen. Ist die Nabelschnur lang und vertrocknet, weist sie Fetzen der 
Placenta auf, so bedürte es einer sehr großen Anstrengung von seiten des Jungen und es brauchte sehr lange Zeit, in unserem Falle 4 Stunden und 20 Minuten, diese abzureißen. Fehlen an der Nabelschnur die Placentafetzen, so ging der Vorgang des Abreißens etwas leichter vor sich und es dauerte, wie die erste Beobachtung zeigte, auch nicht so lange (21/2 Stunden). Dagegen dürften die kurzen, dicken und wenig vertrockneten Nabelschnüre ein Beweis dafür sein, daß diese leicht rissen und das Abreißen auch nur kurze Zeit in Anspruch nahm. Da einige der gefundenen Nabelschnüre die Normallänge sogar erheblich unterschritten, dürfte dies ein Beweis dafür sein, da $\beta$ sie nicht immer an der Placenta abreißen, sondern mitunter auch an einer anderen Stelle. Der in diesem Falle an der Placenta hängende Teil der Nabelschnur wird wohl mit der Nachgeburt aufgefressen, während der andere auf die oben beschriebene Weise auf den Boden gelangt.

Ebenso gestaltete Nabelschnüre fand ich bei Kotanalysen im Kot von Myotis emarginatus (Geoffroy, 1806) und Plecotus auritus (Linnaeus, 1758). Dies berechtigt zu der Annahme, daß die Jungen dieser Arten nach dem Ausstoßen sich ebenso verhalten wie die von Myotis myotis und die Nabelschnüre auch dort in der geschilderten Weise in die Länge gezogen werden.

\section{d. Verhalten des Muttertieres bei Totgeburten.}

Bei Totgeburten ändert das Muttertier sein Verhalten. Wenn das tote Junge am Nabelstrang herunterhängt, so versucht die Mutter durch Herumklettern, wobei das Junge sich meist verfängt, die Nabelschnur abzureißen, oder auch die Placenta mit auszustoßen, wie dies Ditrrich bei Nyctalus noctula und wir bei Myotis myotis und Eptesicus serotinus beobachten konnten. Bei diesen Bemühungen erreichte bei letztgenannter Art die Nabelschnur eine Länge von 65 $\mathrm{mm}$. Führt das Herumklettern oder das Laufen auf dem Boden nicht zum Erfolg, so beißen diese Arten nach längeren, fruchtlosen Bemühungen die Nabelschnur in eigener Körpernähe ab. Wie stark das Abreißen der Nabelschnur durch das Junge auch im Verhaltensschema der Mutter verhaftet ist, zeigt das Bemühen, diesen sonst vom Jungen durchgeführten Vorgang des Ziehens durch Eigenbewegungen zu ersetzen. Erst wenn dies nicht gelingt, beißt sie die Nabelschnur bei sich ab. Wahrscheinlich dürfte sich das Ziehen, das sich über die Nabelschnur auf die Placenta auswirkt, positiv auf die Ablösung derselben auswirken.

\section{ZUSAMMENFASSUNG}

1. Bei der Geburt behalten die Weibchen von Myotis myotis und Eptesicus serotinus die typische Hängelage der Fledermäuse, den Kopf nach unten, bei. Sie heften sich jedoch auch mit den Krallen an der Unterlage fest, wodurch der Körper ggf. die Schräglage des Daches bekommt. Hierbei krümmen sie den Schwanz bogenförmig.

2. Das ausgestoßene Junge klettert zwischen dem Körper der Mutter und der Unterlage am Körper derselben zur Brustzitze und saugt sich fest.

3. Es wird von der Mutter eifrig beleckt. Durch seitliches Hängen des Hinterteiles seines Körpers reißt das Junge die Nabelschnur ab, wozu oft längere Zeit und heftiges Zerren notwendig ist.

4. Die vertrocknete Nabelschnur reißt an der Placenta ab. Diese wird kurz darauf ausgestoßen und von der Mutter aufgefressen.

5. Beim Belecken des Jungen beißt die Mutter die Nabelschnur an diesem ab. Sie fällt zu Boden und wird zwischen den Kotkrümchen gefunden.

6. Die Länge der Nabelschnur läßt einen Rückschluß auf die Dauer der Abreißbewegung und auf ein ähnliches Verhalten anderer Arten bei der Geburt $\mathrm{zu}$.

7. Bei einer Totgeburt sucht das Alttier sich dieser durch Umherklettern zu entbinden, gelingt dies nicht, so beißt sie die Nabelschnur bei sich ab.

\section{LITERATUR}

BreHM, 1912: Tierleben, Säugetiere. Leipzig u. Wien. DitTrich, Lothar, 1958: Haltung und Aufzucht von Nyctalus noctula Schreb. Z. f. Säugetierkunde 23.

Eisentraut, M., 1936: Zur Fortpflanzungsbiologie der Fledermäuse, Z. f. Morphologie u. Ókologie 31.

KolB, ANTON, 1953: Biologische Beobachtungen an Fledermäusen. Säugetierk. Mitteilungen Stuttgart 2.

- 1957: Aus der Wochenstube des Mausohrs, Myotis myo- tis. Säugetierk. Mitteilungen Stuttgart.

- 1958: Nabelschnüre zwischen den Kotkrünchen von Fledermäusen. Z. f. Säugetierkunde 23.

STARCK, D., 1957: Uber die Länge der Nabelschnur bei Säugetieren. Zschr. f. Säugetierk. 22.

Wimsatt, William A., 1960: An analysis of parturation in chiroptera, including new observations on Myotis lucifugus. Journal of Mammalogy 41. 